\title{
3-D Monte Carlo-Based Scatter Compensation in Quantitative I-131 SPECT Reconstruction
}

Yuni K. Dewaraja, Member, IEEE, Michael Ljungberg, Member, IEEE, and Jeffrey A. Fessler, Senior Member, IEEE

\begin{abstract}
We have implemented highly accurate Monte Carlo based scatter modeling (MCS) with 3-D ordered subsets expectation maximization (OSEM) reconstruction for I-131 single photon emission computed tomography (SPECT). The scatter is included in the statistical model as an additive term and attenuation and detector response are included in the forward/backprojector. In the present implementation of MCS, a simple multiple window-based estimate is used for the initial iterations and in the later iterations the Monte Carlo estimate is used for several iterations before it is updated. For I-131, MCS was evaluated and compared with triple energy window (TEW) scatter compensation using simulation studies of a mathematical phantom and a clinically realistic voxel-phantom. Even after just two Monte Carlo updates, excellent agreement was found between the MCS estimate and the true scatter distribution. Accuracy and noise of the reconstructed images were superior with MCS compared to TEW. However, the improvement was not large, and in some cases may not justify the large computational requirements of MCS. Furthermore, it was shown that the TEW correction could be improved for most of the targets investigated here by applying a suitably chosen scaling factor to the scatter estimate. Finally clinical application of MCS was demonstrated by applying the method to an I-131 radioimmunotherapy (RIT) patient study.
\end{abstract}

Index Terms-I-131 SPECT, image reconstruction, Monte Carlo, scatter correction, SPECT quantification.

\section{INTRODUCTION}

$\mathbf{T}$ HE loss of SPECT image quality and quantification accuracy due to photon scatter is well known. The various scatter compensation techniques developed in the past have been reviewed recently [1]. These methods can be grouped in to two broad categories: subtraction-based and reconstruction-based scatter compensation. In the subtraction-based approach the scatter component is estimated and subtracted from the projection data prior to reconstruction. The scatter estimate is typically obtained from multiple energy window acquisitions. In the reconstruction-based approach the scatter is included in the statistical model. With this approach there is no explicit subtraction of scatter counts, hence the noise increase

Manuscript received November 1, 2004. This work was supported in part by the National Institutes of Health, United States Department of Health and Human Services, under Grant R01 EB001994, and in part by the National Science Foundation (for computing resources provided by the National Partnership for Advanced Computational Infrastructure at the San Diego Supercomputer Center) under cooperative agreement ACI-9619020.

Y. K. Dewaraja is with the Department of Radiology, University of Michigan, Ann Arbor, MI 48109-0552 USA (e-mail: yuni@umich.edu).

M. Ljungberg is with the Department of Medical Radiation Physics, Lund University, Lund, Sweden (e-mail: michael.ljungberg@ radfys.lu.se).

J. A. Fessler is with the Department of Electrical Engineering and Computer Science, University of Michigan, Ann Arbor, MI 48109 USA (e-mail: fessler@eecs.umich.edu).

Digital Object Identifier 10.1109/TNS.2005.862956 associated with scatter subtraction methods is avoided. Comparative studies have shown that iterative reconstruction with accurate modeling of scatter is superior to pre-reconstruction scatter subtraction [2], [3].

Monte Carlo photon transport is a general method that can be used for accurate scatter modeling without the need for empirical approximations or multi-window acquisitions. In the past Monte Carlo simulation has been used for scatter modeling in 2D (Floyd et al. [4]) and 3D (Beekman et al. [5], Cot et al. [6]) iterative SPECT reconstruction. The method of Floyd et al. has massive computational requirements for pre-calculating and storing the full system matrix. Hence it was only implemented for 2D SPECT. Recently, because of computing advances the practical feasibility of this approach for 3D SPECT was re-investigated [7]. In the method of Beekman et al. the scatter-modeling step involves the Monte Carlo calculation of low noise scatter projections. They developed a highly efficient Monte Carlo simulator to be used as the projector in the iterative reconstruction algorithms. Their evaluations for Tc- $99 \mathrm{~m}$ imaging demonstrated that the reconstructions based on the Monte Carlo projector are superior to those based on an advanced analytical scatter model.

Our interest is in tumor and organ activity quantification for dosimetry in patients undergoing I-131 RIT for Non-Hodgkin's lymphoma [8]. The goal of the present work was to implement highly accurate Monte Carlo based scatter modeling with 3-D OSEM reconstruction for I-131 SPECT. In I-131 SPECT, both object scatter and collimator scatter is highly significant because of downscatter from the multiple gamma ray emissions (637 kev, $722 \mathrm{kev}$ ) at higher energies than the $364 \mathrm{kev}$ emission typically used for imaging. The Monte Carlo projector used in the present work, SIMIND [9], includes accurate physical modeling of the collimator (including collimator scatter and penetration) and has been extensively validated for I- 131 by comparing with measured data [10], [11].

The proposed scatter correction can be applied to clinical studies if the patient's measured activity distribution and co-registered attenuation map are available to define the input object to the Monte Carlo projector. In the past when SPECT studies were performed on RIT patients at our clinic, co-registered x-ray CT derived attenuation maps were used for attenuation correction [8]. Hence, SPECT derived activity maps and the attenuation maps are readily available for the Monte Carlo based scatter correction. In such cases and with the recent availability of dual modality SPECT-CT cameras, there is much incentive to carry out the Monte Carlo based scatter compensation. Although MCS has the potential for highly accurate scatter estimation it has high computational requirements to generate low noise 
scatter projections. In addition, this correction cannot account for scattered photons that originate outside the SPECT camera field of view (FOV) because the input object to the Monte Carlo projector is limited by the camera FOV. Simpler methods based on multiple-energy window acquisitions can potentially correct for these scattered photons that originate outside the FOV.

In the present implementation of MCS, a triple energy window scatter correction (TEW) [12] is used for the initial iterations. In the later iterations, the Monte Carlo scatter estimate is held constant for several iterations before it is updated. A similar approach was reported by Kadrmas et al., which they called intermittent reconstruction based scatter compensation [13]. Their work in Tc-99m SPECT showed that the projected scatter estimates nearly fully converge after only 2 or 3 iterations of OSEM. Hence the scatter estimate was held constant for the remaining iterations, thereby accelerating the reconstruction process by limiting the number of iterations during which scatter is modeled. In a more recent implementation of reconstruction based scatter compensation the scatter estimate was updated at each iteration using a highly efficient Monte Carlo simulator [5].

In this paper we discuss the implementation of MCS and carry out phantom studies to evaluate the method and compare it with TEW scatter correction used thus far in our clinical SPECT studies for I-131 RIT. Finally MCS is applied to a patient study to demonstrate clinical application. The present study does not focus on methods to speed-up the Monte Carlo projector or evaluate errors in the scatter model due to mis-registration and noisy attenuation maps.

\section{METHODS}

\section{A. Reconstruction}

In our implementation of both TEW and MCS, the scatter estimate is included in the OSEM algorithm in a manner appropriate for Poisson statistics [14], i.e., scatter is included in the statistical model as a "known" additive term as shown below:

$$
Y_{i} \approx \text { Poisson }\left\{\sum_{j=1}^{n} g_{i j} x_{j}+s_{i}\right\}
$$

where $\mathrm{x}$ is the unknown image consisting of $\mathrm{n}$ voxels, $\mathrm{Y}$ is the measured projection data, $g$ is the system matrix excluding scatter, and s is the TEW or Monte Carlo scatter estimate. With scatter treated in this manner we include only attenuation and 3D depth dependent detector response in the forward/backprojector. This approach avoids the massive computational requirements associated with including scatter in the system matrix. Reconstruction was carried out with an unregularized multiplane (3D) OSEM algorithm using 6 subsets.

In the TEW correction the same scatter estimate is used in all the iterations. In this method, the photopeak window scatter counts for each pixel is estimated from counts acquired in two narrow sub-windows. The sub-window location was chosen to be adjacent to the photopeak window based on our previous work characterizing the energy distribution of I-131 scatter [10]. The sub-window projection data is first filtered because the narrow-energy windows will result in a noisy estimate. To determine the optimum filter both Gaussian and Butterworth fil- ters were evaluated and based on these studies a Gaussian filter with 3 pixel FWHM was selected for filtering the sub-window data in the present study. A previous study by another group investigated the filtering of TEW scatter estimates in ordered subsets reconstructions [15].

For the MCS correction, the TEW scatter estimate is used in the initial iterations (specifically first 20 iterations in the present study), until a reasonable reconstructed image is obtained. When generating the first MCS scatter estimate this TEW corrected reconstruction together with the phantom/patient attenuation map defines the input object to the SIMIND projector. Gaussian smoothing ( $\mathrm{FWHM}=3$ pixels) is also performed on the SIMIND generated scatter estimate. To reduce noise, an analytical projector is used for the primary photons. In our implementation the Monte Carlo scatter estimate is generated one time and is used for multiple iterations before recalculating a new scatter estimate based on the latest reconstruction. In order to determine how many updates of the Monte Carlo scatter estimate are necessary, we compared using 2 updates with using 6 updates. With two updates, the Monte Carlo scatter estimates were generated after iterations 20 and 40 while with six updates they were generated after iterations $20,24,28,32,36$, and 40 . In the iterations in between these updates the scatter estimate was held constant.

To investigate the number of photon histories needed to minimize noise we compared using $10^{7}, 10^{8}$, and $10^{9}$ photons per projection (at each angle) when generating the Monte Carlo scatter estimate. The total run time for all projections with $10^{7}$ and $10^{8}$ histories was 2 hours and 20 hours respectively on a DEC ALPHA 1000 workstation. The simulation with $10^{9}$ histories was carried out on multiple processors of the IBM POWER4+ system at the San Diego Supercomputer Center, University of California.

\section{B. Phantom Simulations}

For the phantom studies, Monte Carlo simulation was used not only to generate the scatter estimate during reconstruction but also to obtain the original projections. For the original phantom projections a large number $\left(10^{10}\right.$ photons/projection $)$ of photon histories were simulated to generate essentially noise free projection data. Note that different random number seeds were used when generating the original projection set and when generating the scatter estimates during the reconstruction, hence the data sets are not correlated.

Both a mathematical elliptical phantom and the anthropomorphic voxel-man phantom [16] were used in the present study. For both phantoms, the distance from the phantom center to the camera was $26 \mathrm{~cm}$. The elliptical tank was $23 \times 31.5 \mathrm{~cm}$ and $20.5 \mathrm{~cm}$ in height and contained 2 hot-spheres $(5.8 \mathrm{~cm}$, and $2.9 \mathrm{~cm}$ diameter), one cold-sphere (5.8 $\mathrm{cm}$ diameter) and one warm-sphere $(5.8 \mathrm{~cm}$ diameter) in a uniform background. The sphere to background activity concentration ratio for the hot spheres was 5:1 while that for the warm sphere was 1:2. The entire elliptical phantom was within the SPECT camera FOV. The voxel-man phantom was created from X-ray CT slices of a living adult male. All major internal structures have been segmented and arbitrary mass density and activity values can be assigned to the voxels within each structure. Here we assigned the 
following activity concentration ratios to the structures to reflect a typical situation in SPECT imaging after the therapy administration of I-131 [17]: kidney, 80; liver, 28; lung, 28; spleen, 52; blood-pool, 48; rest of the body, 4. To mimic the situation in patient imaging all 240 slices of the torso phantom were simulated, although only 60 of these slices were within the axial FOV of the SPECT camera, which is $24 \mathrm{~cm}$. It is important to include slices outside the FOV since photons originating outside the camera FOV that undergo scatter can contribute to the image.

A Picker Prism 3000 SPECT camera was modeled with a commercial ultra-high energy collimator (UHE) that minimizes septal penetration. Previously we have validated the SIMIND code with measured data from this camera system [10]. The present version of SIMIND allows for a hexagonal hole shape collimator with explicit modeling of collimator scatter and penetration [18]. The hole size, septa and thickness of the UHE collimator were $5.08 \mathrm{~mm}, 3.43 \mathrm{~mm}$, and $77 \mathrm{~mm}$. The camera model in the simulation included a $5 \mathrm{~cm}$ glass layer behind the $\mathrm{NaI}$ crystal to model backscatter. Studies have shown that such a layer can be effective in modeling backscatter since explicit modeling of all structures behind the crystal is not practical [10], [19]. Multiple (four) scatter orders were used for scatter in both the object and the camera. The energy resolution at $364 \mathrm{keV}$ was $10.2 \%$ (FWHM). SPECT simulations employed 360 degrees, 60 angles a $20 \%$ photopeak at $364 \mathrm{keV}$, and two $6 \%$ adjacent scatter correction windows at $318 \mathrm{keV}$ and $413 \mathrm{keV}$. For the elliptical phantom the matrix size was $64 \times 64$ with a pixel size of $7.2 \mathrm{~mm}$ while for the voxel-man the matrix was $128 \times 128$ with a pixel size of $4 \mathrm{~mm}$.

\section{Evaluation}

In addition to visual comparison of images and profiles several image quality measures were calculated to quantitatively evaluate the scatter compensation. The normalized mean square error (NMSE) defined below was calculated for both the scatter projections and for reconstructed images while the other measures defined below were calculated for reconstructed images only. To evaluate the TEW and MCS estimated scatter projections, the true scatter projections were obtained by separately tracking scatter events in the simulation that generated the original phantom projection data. To evaluate the reconstructed images, projection data was reconstructed 1) without scatter compensation 2) with TEW compensation 3) and with MCS compensation. The "ideal" projection data set was also generated by separately tracking only the primary (scatter-free) photons in the simulation and this data set was reconstructed without scatter compensation. All the above reconstructions included attenuation correction and 3D depth detector response modeling. The true boundaries in the phantom were used to define target volumes of interest (VOIs). This mimicked the situation in clinical I-131 SPECT studies at our clinic where co-registered CT images are used to define tight tumor boundaries.

For reconstructed images, the image quality measures (NMSE and bias) were calculated relative to the defined activity map of the "true" phantom. A calibration factor was used to convert the reconstructed image from counts to activity, before calculating these image quality measures. The calibration factor was determined by simulating a measurement with a known activity point source in air and the same camera system used for the phantom imaging. When image quality measures in the reconstruction are calculated relative to the "true" phantom, it is difficult to separate scatter effects from partial volume effects (PVE). The PVE are caused by spread or blurring of regional counts to surrounding area due to the finite spatial resolution of the system and due to collimator penetration. The "spill-out" of counts from the target to the background decreases the counts in the target while the "spill-in" of counts from the background to the target increases the target counts.

The accuracy was evaluated using the NMSE defined by

$$
\% \mathrm{NMSE}=100 *\left(\sum_{i}\left(x_{i}-p_{i}\right)^{2}\right) / \sum_{i} p_{i}^{2}
$$

where the sums are over a VOI or the total image. NMSE is computed for the noise free images. When the NMSE is calculated for phantom projection data, $\mathrm{x}$ represents the estimated scatter projection and $\mathrm{p}$ represents the true scatter projection. When NMSE is calculated for reconstructed data, $\mathrm{x}$ represents the reconstructed image and p represents the "true" phantom.

For patient projection data where the true scatter projection is not known, (2) was used to calculate the normalized mean square difference (\%NMSD) between succesive Monte Carlo generated scatter updates. In this case, $x$ represents the previous MCS estimate and $\mathrm{p}$ the present MCS estimate.

Since our task is activity quantification in tumor and organs we also calculated the bias in the target activity defined as

$$
\% \text { bias }=100 *\left(\sum_{i} x_{i}-\sum_{i} p_{i}\right) / \sum_{i} p_{i}
$$

where the sums are over the target VOI and $\mathrm{x}$ represents the reconstructed image and p represents the "true" phantom. The bias is computed for the noise free images.

To measure noise, the original phantom projection data was scaled to 20 million total counts (this corresponds to a typical patient therapy scan in RIT) before the addition of Poisson distributed noise. The noise-free projection data and the noisy projection data were both reconstructed as described previously. The noise was measured using the normalized standard deviation (NSD) as defined by Frey et al. [2]

$$
\mathrm{NSD}=\sqrt{\sum_{i}\left(x_{i}-\bar{x}_{i}\right)^{2} /(N-1)}\left(\sum_{i} \frac{\bar{x}_{i}}{N}\right)^{-1}
$$

where the sums are over a VOI or the total image. Here, $\mathrm{x}$ is the image reconstructed from noisy data, $\bar{x}$ is the image reconstructed from noise free data and $\mathrm{N}$ is the number of pixels.

\section{Patient Study}

To demonstrate clinical applicability the MCS compensation was applied to SPECT data for one patient who had undergone imaging following RIT at our clinic. The lymphoma patient had been imaged 44 hours after therapy administration of $4 \mathrm{Gbq}$ of I-131 tositumomab using the same SPECT camera and collimator modeled in the present phantom studies. The SPECT matrix size was $64 \times 64$ with a pixel size of $7.2 \mathrm{~mm}$. The co-registered CT-derived attenuation map was available as it had been used for SPECT attenuation correction. 
TABLE I

\%NMSE FOR THE SCATTER ESTIMATES OF THE ELLIPTICAL PHANTOM

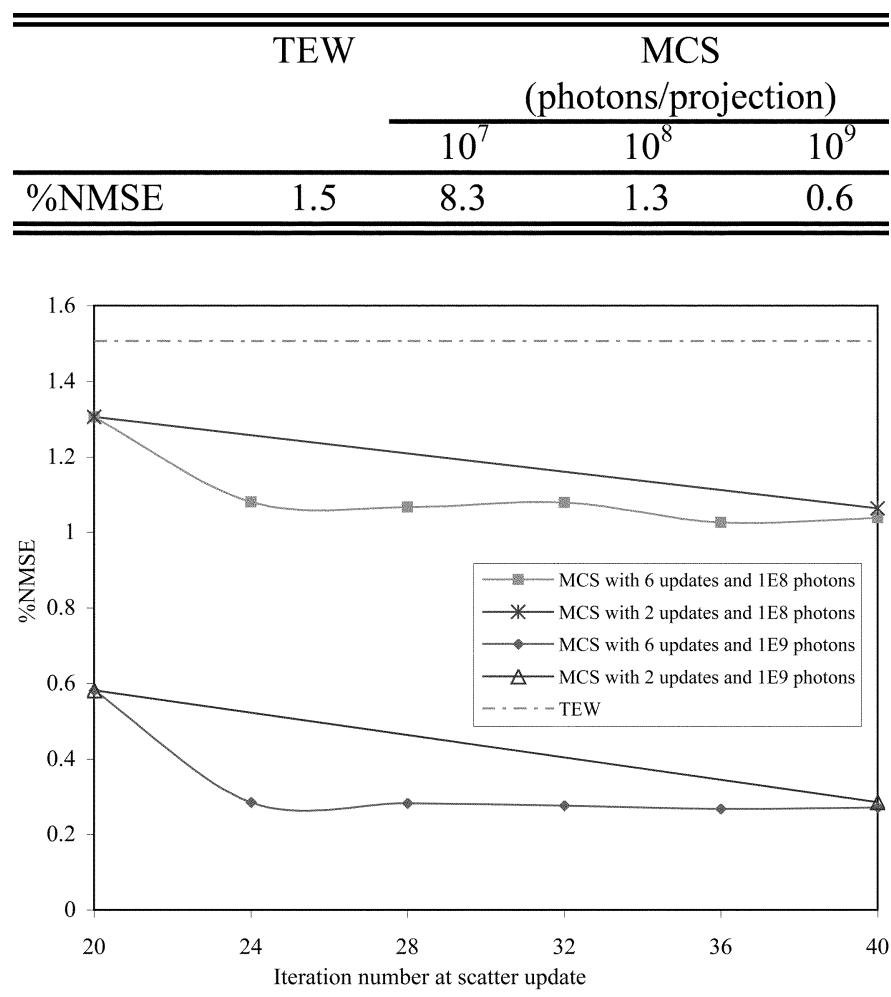

Fig. 1. \%NMSE as a function of the iteration number for the elliptical phantom scatter projections.

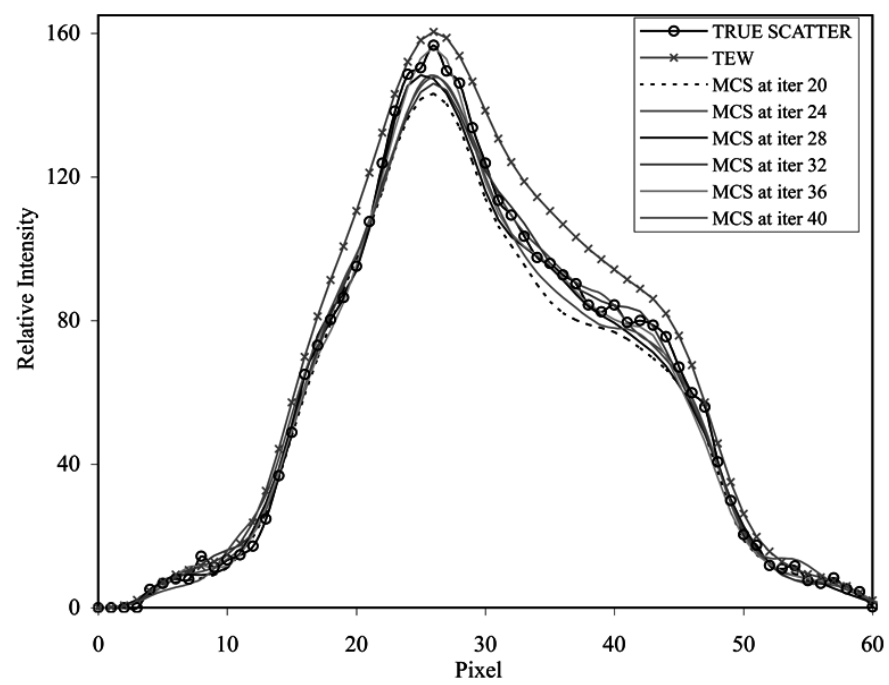

Fig. 2. Profile across a typical scatter projection of the elliptical phantom.

\section{RESULTS AND DISCUSSION}

\section{A. Elliptical Phantom}

1) Scatter Projections: As discussed in the methods, for projection data the NMSE was calculated between the estimated scatter projections and the true scatter projection. The NMSEs for the TEW estimate and the first MCS estimate with different numbers of photon histories are compared in Table I. Based on these results it is evident that $10^{7}$ photons/projection is too few histories for generating an accurate Monte Carlo

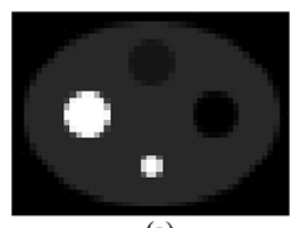

(a)

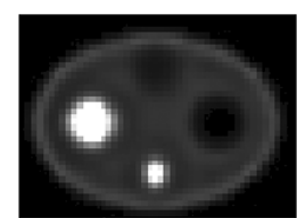

(d)

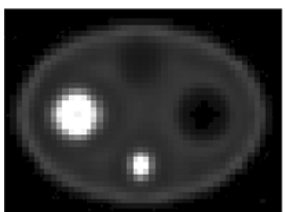

(b)

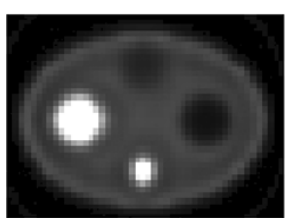

(c)

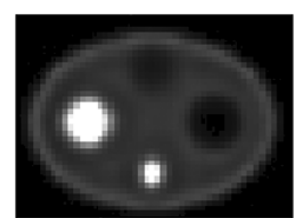

(e)
Fig. 3. Slice of elliptical phantom: (a) activity map; (b) reconstructed image corresponding to primary photons only; (c) image reconstructed without scatter correction; (d) image reconstructed with TEW; (e) image reconstructed with MCS.

scatter estimate. With both $10^{8}$ and $10^{9}$ photons/projection the NMSE values for MCS are better than that for TEW.

Next we evaluated the scatter estimates with just two Monte Carlo updates (after iteration 20 and 40) and with six updates (after iteration 20, 24, 28, 32, 36, 40). For both cases the NMSE in the estimated scatter projections is plotted as a function of the iteration number in Fig. 1. Results are shown for both $10^{8}$ and $10^{9}$ photons/projection. For comparison the NMSE for the TEW estimate is also shown, but this estimate is generated only once, hence the NMSE remains constant with iteration. According to these results there is a clear improvement when going from the first MCS update to the second, however after that there is not much to be gained by further updating the MCS estimate. In fact, the NMSE is almost unchanged at iteration 40 irrespective of whether 2 or 6 updates were used. The plot also shows that the NMSE for the MCS estimate is always superior to the NMSE for the TEW estimate. Fig. 2 shows profiles across a typical projection for the TEW and Monte Carlo scatter estimates and the true scatter. According to this profile, the TEW method overestimates the scatter and the first MCS estimate underestimates the scatter. MCS underestimates the scatter because the TEW estimate is used in the initial iterations before generating the first MCS update. An overestimation of scatter by TEW leads to a reconstructed image with too few counts. Since this image is used as the input to the Monte Carlo projector, the first MCS estimate will be too low. After the first MCS update, the profiles for the next five MCS updates that follow are almost indistinguishable from one another and are in good agreement with the true scatter profile. This is consistent with our results for the NMSE in Fig. 1, where it was shown that no significant change was seen after the second MCS update. Note that in Fig. 2, the profiles corresponding to the scatter estimates are smoother than that corresponding to the true scatter because as described previously Gaussian smoothing was applied to the estimates.

2) Reconstructed Images: The above evaluations of the scatter projections showed that the MCS estimate converge after just two updates. Therefore for the evaluations of the reconstructed images we only consider the case where two MCS updates (after iteration 20 and 40) were used in the reconstruction. We also only consider the case where $10^{9}$ photons/projection were used when generating the estimate. 
TABLE II

Minimum (Over All Iterations) \%NMSE And \%Bias For the Different ReConstructions of the Two Phantoms

\begin{tabular}{lcccccccc}
\hline \hline & \multicolumn{2}{c}{ Primary } & \multicolumn{2}{c}{ No correction } & \multicolumn{2}{c}{ TEW } & \multicolumn{2}{c}{ MCS } \\
& \%NMSE & \%bias & \%NMSE & \%bias & \%NMSE & \%bias & \%NMSE & \%bias \\
\hline Elliptical phantom & & & & & & & & \\
\hline Total image & 9.3 & -0.7 & 23.0 & 37.1 & 9.9 & -5.1 & 9.5 & -1.1 \\
Large hot sphere & 9.0 & -10.4 & 12.0 & 11.2 & 10.3 & -8.6 & 9.1 & -7.6 \\
Small hot sphere & 15.0 & -23.0 & 8.4 & -8.6 & 15.2 & -24.3 & 13.7 & -23.1 \\
Warm sphere & 9.4 & 11.9 & 82.1 & $\mathbf{8 4 . 0}$ & 8.6 & -0.2 & 9.1 & 7.6 \\
& & & & & & & & \\
Voxel-man phantom & & & & & & & & \\
\hline Total image & 15.1 & 1.3 & 32.2 & 52.2 & 15.9 & -4.1 & 15.4 & 2.3 \\
Liver & 5.7 & -7.4 & 22.0 & 36.1 & 6.6 & -11.5 & 5.5 & -2.4 \\
Lung & 6.0 & -2.2 & 12.4 & 24.5 & 6.8 & -3.0 & 6.1 & 1.2 \\
Kidney & 11.8 & -11.1 & 12.8 & 12.4 & 13.0 & -11.4 & 11.7 & -9.0 \\
Spleen & 8.9 & -9.9 & 12.5 & 17.6 & 10.5 & -11.8 & 8.4 & -7.2 \\
\hline \hline
\end{tabular}

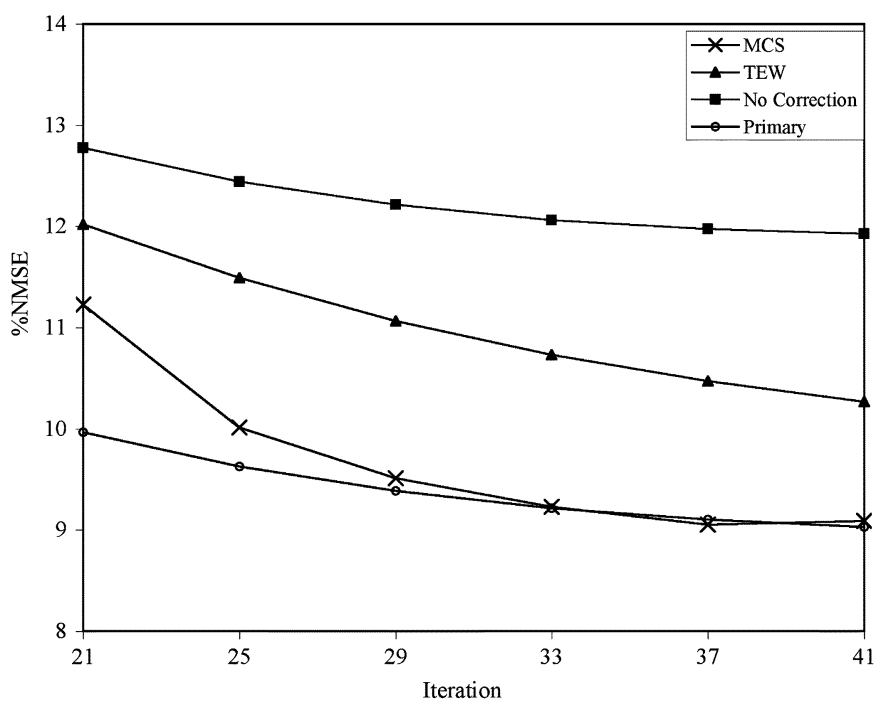

Fig. 4. \%NMSE as a function of the iteration number for the large hot sphere ROI.

A typical slice of the elliptical phantom defined activity map and the reconstructions at iteration 41 are shown in Fig. 3. Both the TEW and MCS corrected images look very similar to the image corresponding to the primary (scatter-free) data. As discussed in the methods section, for the reconstructed images the NMSE and bias were calculated relative to the true phantom. For the large hot sphere, the NMSE is plotted as a function of iteration for the different reconstructions in Fig. 4. Note that we begin the plot at iteration 21 because in our implementation TEW and MCS methods are the same for the first 20 iterations. The NMSE initially decrease rapidly with iteration, but then decreases more slowly or increases with iteration due to noise. For the different reconstructed images the minimum (over all iterations) \%NMSE and \%bias values for the total image and the sphere ROIs are given in Table II. The results of Table II and Fig. 4 shows that both scatter modeling methods improve the NMSE and bias when compared with reconstruction without scatter correction. The only exception is for the small hot sphere where partial volume effects dominate. As discussed in the methods section when image quality measures are calculated relative to the "true" phantom, not only scatter effects but also partial volume effects are included in the evaluation. The loss of counts in a target due to PVE can potentially be "compensated" by uncorrected scatter counts. For the small hot sphere ROI the loss of counts due to "spill-out" is partially "compensated" by scatter counts, hence the image without scatter correction can produce better NMSE and bias values than the scatter corrected images or even the primary image. According to the results of Table II the scatter correction has the most impact on the warm sphere. For example, the bias for the warm sphere without scatter correction is $84 \%$, but decreases to $7.6 \%$ with MCS correction. For the warm sphere, since the target is relatively large and background activity is higher than the target activity, the dominant PVE will be "spill-in." Since scatter counts cannot compensate for "spill-in" counts, the error without scatter correction is very large. For the warm sphere, the TEW results are superior to the MCS results possibly because TEW overcorrects for scatter thereby "compensating" for "spill-in." For all other cases in Table II, the NMSE and bias values for MCS are superior to those for TEW, however the improvement is not very large. The bias results for the total image, where PVE do not play a role, demonstrate that the MCS correction is more accurate than the TEW correction.

NSD is plotted as a function of iteration for the different reconstructions in Fig. 5. The upper set of curves corresponds to the total image and the lower set to the large hot sphere ROI. The NSD values for MCS are slightly lower than those for TEW.

\section{B. Voxel-Man Phantom}

1) Scatter Projections: For the voxel phantom, Monte Carlo scatter estimates were generated with $10^{9}$ photons/projection. As with the elliptical phantom, the TEW estimate was used for the first twenty OSEM iterations and we compared using just two updates (after iteration 20 and 40) and six updates (after iteration 20, 24, 28, 32, 36, 40) of the Monte Carlo scatter estimate. For both cases the NMSE in the estimated scatter projections is plotted as a function of the iteration number in Fig. 6. As for the elliptical phantom, there is a clear improvement when going from the first MCS update to the second, however after that there is not much to be gained by further updating the MCS estimate. Fig. 7 shows the profiles across a typical scatter projection. As for the case with the elliptical phantom, the profiles show that TEW overestimates the scatter and the first MCS estimate underestimates the scatter. There is excellent agreement between 


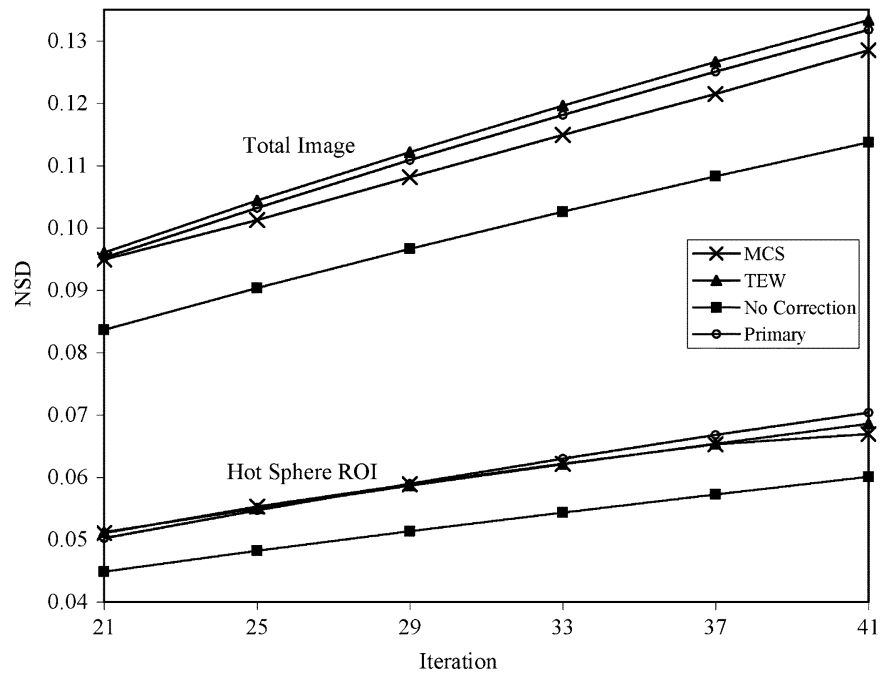

Fig. 5. NSD as a function of the iteration number for the elliptical phantom. Upper set of curves corresponds to the total image and the lower set of curves corresponds to the large hot sphere ROI.

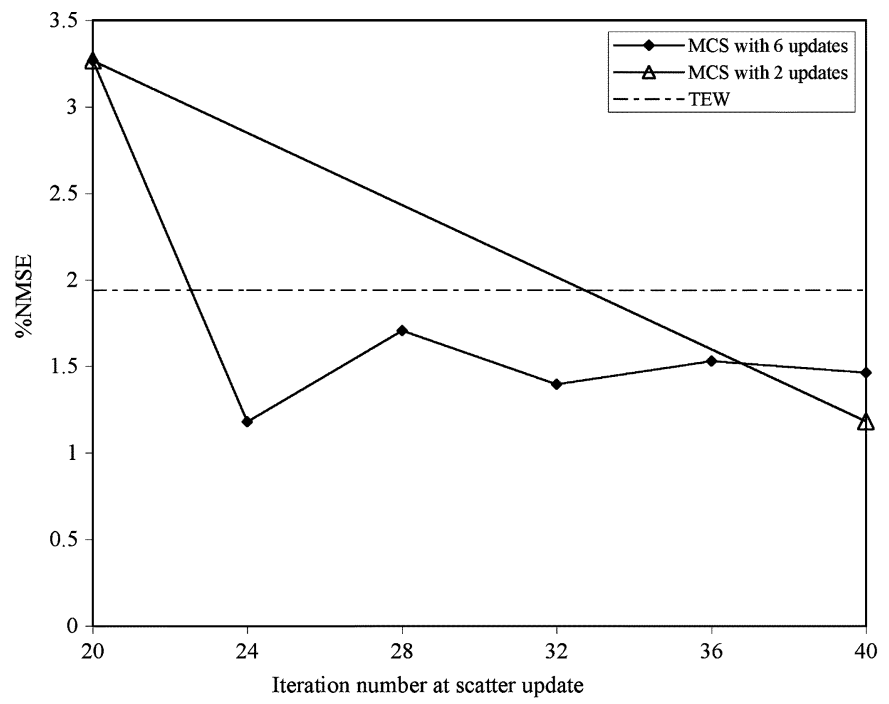

Fig. 6. \%NMSE as a function of the iteration number for the voxel-man phantom scatter projections.

the second Monte Carlo scatter estimate and the true scatter profile. Note that the profiles corresponding to the scatter estimates are smoother than that corresponding to the true scatter because Gaussian smoothing was applied to the estimates.

2) Reconstructed Images: As for the elliptical phantom, the above evaluations for the voxel phantom showed that the Monte Carlo estimated scatter projections converge after just two updates. Therefore for the evaluations of the reconstructed images we only consider the case where two MCS updates (after iteration 20 and 40) were used in the reconstruction.

Fig. 8 shows one slice of the voxel phantom activity and attenuation maps and the reconstructed images at iteration 41 . The figure shows the degradation in contrast when no scatter correction is used. Both the TEW and MCS corrected images look very similar to the image corresponding to the primary (scatter-free) data. For the total image and organs the minimum (over all iterations) \%NMSE and \%bias values are given in Table II. In almost all cases both TEW and MCS compensation improves the

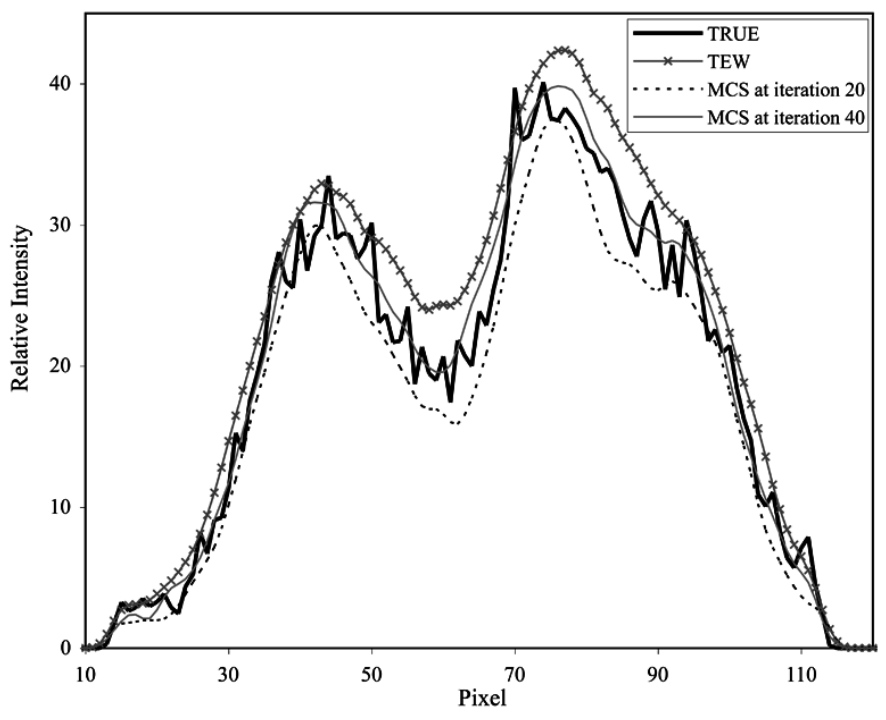

Fig. 7. Profile across a typical scatter projection of the voxel-man phantom.

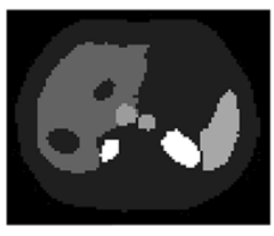

(a)

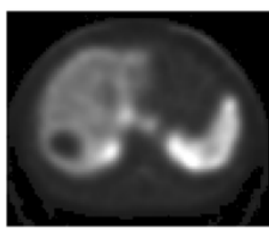

(d)

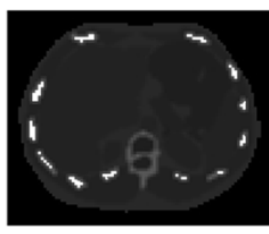

(b)

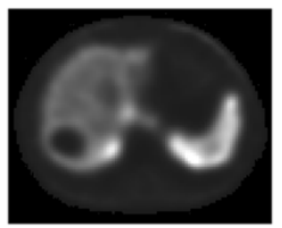

(e)

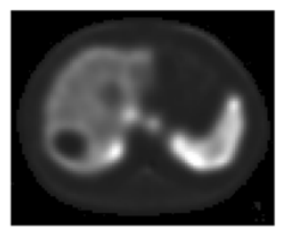

(c)

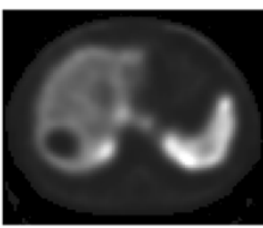

(f)
Fig. 8. Slice of voxel-man phantom: (a) activity map; (b) attenuation map; (c) reconstructed image corresponding to primary photons only; (d) image reconstructed without scatter correction; (e) image reconstructed with TEW; (f) image reconstructed with MCS.

TABLE III

MiNIMUM (OVER All ITERATIONS) \%NMSE AND \%BIAS FOR RECONSTRUCTION With THE SCALED TEW SCATTER ESTIMATE

\begin{tabular}{lcc}
\hline \hline & \multicolumn{2}{c}{ TEW with scaling } \\
\cline { 2 - 3 } & \%NMSE & \%bias \\
\hline Elliptical phantom & & \\
Total image & 9.7 & -0.9 \\
Large hot sphere & 10.0 & -6.5 \\
Small hot sphere & 14.3 & -22.7 \\
Warm sphere & 9.3 & 6.6 \\
& & \\
Voxel-man phantom & & \\
\hline Total image & 15.9 & 1.4 \\
Liver & 5.9 & -6.7 \\
Lung & 6.5 & -0.06 \\
Kidney & 12.3 & -8.6 \\
Spleen & 9.7 & -8.8 \\
\hline \hline
\end{tabular}

NMSE and bias compared with images reconstructed without scatter correction. The improvement is especially significant for the total image and large organs such as the liver and lung, where PVE are small. Note that the kidney though large in volume has 

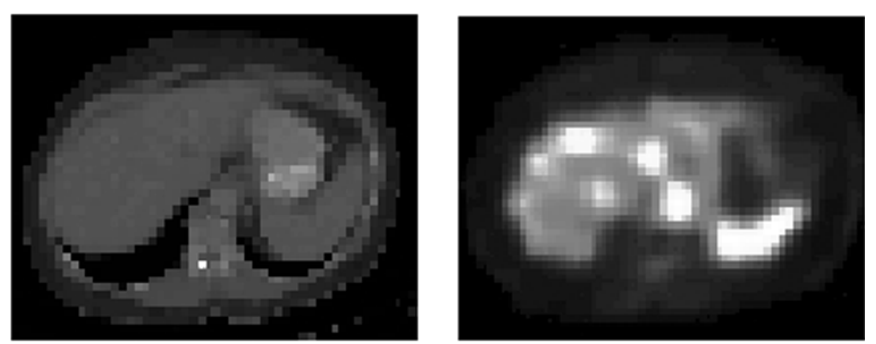

Fig. 9. One slice of the patient's CT-derived attenuation map and co-registered SPECT activity distribution used as input to the Monte Carlo projector.

a hollow region near its center and PVE can be significant for this organ. In Table II, all of the NMSE and bias values for MCS are superior to those for TEW, but in some cases the improvement is not large.

\section{TEW With Scaling}

The profiles of Figs. 2 and 7 show that TEW overestimates scatter in both the elliptical phantom and the voxel-man phantom. This is also evident in Table II where all of the \%bias results for TEW are in the negative direction. Based on these results we investigated scaling the TEW scatter estimate by a factor slightly less than one. For different values of the scale factor (in the range 0.8 to 1 ) the \%NMSE between the estimated scatter projections and the true scatter projections was calculated over all projections as defined in (2). A scale factor of 0.9 resulted in the minimum \%NMSE for both the elliptical phantom and the voxel-man phantom. Therefore, the TEW scatter estimates were scaled by 0.9 and the reconstructions with the TEW correction were repeated for the two phantoms. New \%bias and \%NMSE values were calculated for the reconstructed images (Table III). Comparing results of Tables II and III shows that in almost all cases the results for TEW with scaling are slightly superior to the results for conventional TEW. The warm sphere is an exception because here the dominant PVE is "spill-in" and the overcorrection by conventional TEW compensates for "spill-in" counts. For both phantoms, the \%bias and \%NMSE results for TEW with scaling are very similar to the MCS results.

Here we used a constant scale factor for both phantoms and for all pixels in the projections. However in practice, the required scaling may depend on the object and the activity distribution ("hot' versus "cold" regions, for example). Hence, determining an optimum scale factor may not always be easy.

\section{Patient Study}

Patient data was reconstructed using the TEW scatter estimate for the first 20 iterations. The SPECT reconstruction at iteration 20 and the patient attenuation map defined the input object to the Monte Carlo projector (a typical slice of the input object is shown in Fig. 9). As in the voxel phantom study MCS estimates were generated using $10^{9}$ photons/projection. We again evaluated using up to six updates of the MCS estimate. In the case of patient data since the true scatter projection is not known, the change in succesive scatter updates was evaluated. Using (2) the normalized mean square difference was calculated between the

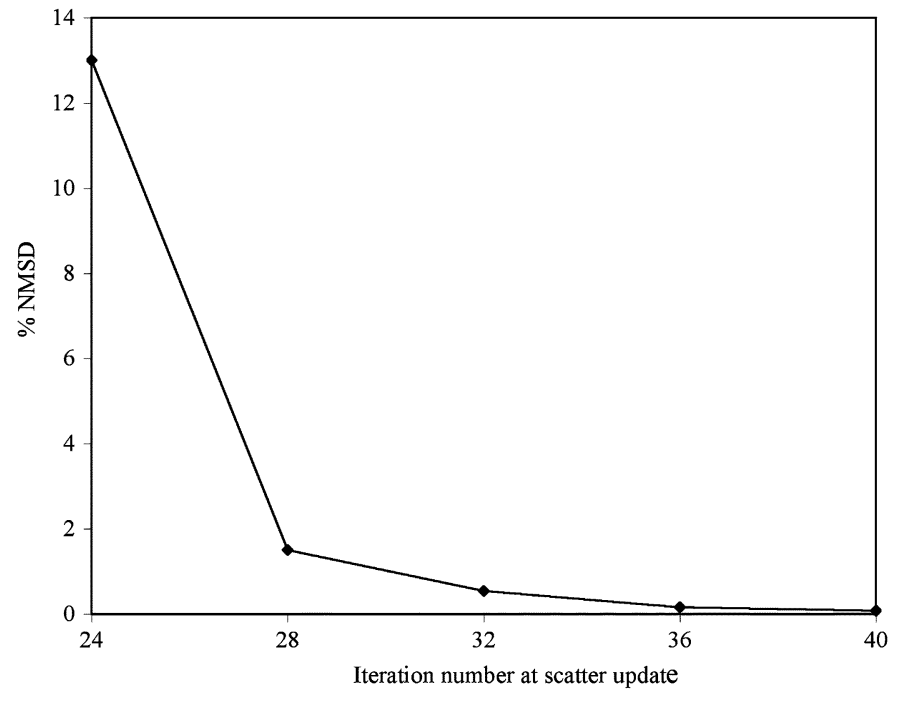

Fig. 10. \%NMSD as a function of iteration number for the patient MCS projections.

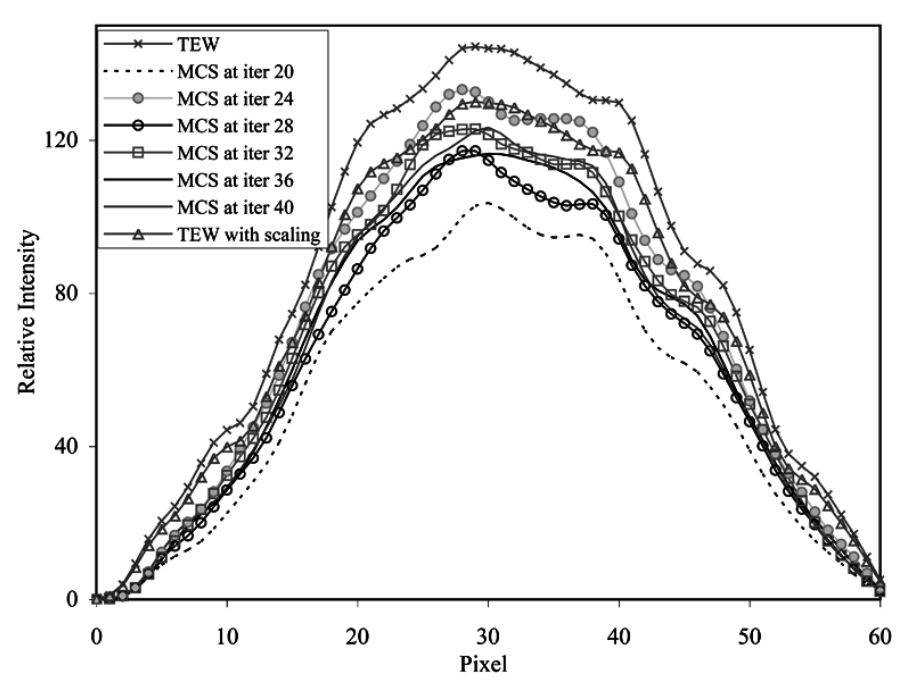

Fig. 11. Profile across a typical scatter projection for the patient data.

current MCS estimate and the previous MCS estimate (Fig. 10). According to the figure, only 2 and possibly 3 MCS updates are needed for the patient data because the difference between the estimates diminishes after that. The NMSD between the first and second update is $13 \%$ but is only $1.5 \%$ between the second and third update and $0.5 \%$ between the third and fourth update. Fig. 11 shows profiles across a typical scatter projection. For the patient the true scatter projection is not known, but as in the phantom studies TEW again overestimates scatter compared with MCS. However, when the 0.9 scaling factor, optimized previously for the phantom studies, is used here the scaled TEW scatter estimate agrees closely with MCS.

Our results for both phantom and patient MCS scatter projections are consistent with the previously discussed intermittent reconstruction based scatter compensation implemented by Kadrmas et al. [13]. As in the present I-131 SPECT study, their study for Tc-99m showed that the projected scatter estimates nearly fully converge after only 2 or 3 iterations of OSEM. 


\section{CONCLUSION}

These studies demonstrate highly accurate scatter compensation with MCS, even with just two updates of the Monte Carlo estimate. The phantom studies showed that the NMSE for Monte Carlo generated scatter estimates with both $10^{8}$ and $10^{9}$ photons/projection were superior to the NMSE for the TEW scatter estimate. Increasing the number of MCS updates from one to two showed a significant decrease in NMSE, but further updates did not lead to further improvement. In general, the accuracy and noise of the images reconstructed with MCS were superior to images reconstructed with TEW. However, the improvement was not large, and in some cases may not justify the large computational requirements of MCS. In this investigation, the same scatter model was used to generate the projection data and to perform image reconstruction. Despite these favorable conditions, the benefit of the MCS method relative to the TEW method was modest in the present I-131 studies. In practice there can be mismatch between the "true" system model and the model used for image reconstruction, even when a validated Monte Carlo algorithm is used. Such model mismatch might further reduce the benefits of the MCS approach relative to simpler methods like TEW. Furthermore, it was shown that the TEW correction could be improved for most of the targets investigated here by applying a suitably chosen scaling factor to the scatter estimate.

Clinical applicability of the method was demonstrated by the patient study, where as in the phantom studies TEW overestimated the scatter compared with MCS and the MCS estimate approached convergence after just 2-3 updates. The SIMIND simulation time to generate the scatter estimate was relatively long (20 hours on a workstation for $10^{8}$ photons/projection). However, the focus of the present study was not on accelerating the calculation, but rather on the implementation and evaluation. Furthermore, since in the present implementation the MCS estimate is not updated at each iteration the speed of the Monte Carlo simulator is less important than in implementations where the estimate is updated at each iteration.

\section{REFERENCES}

[1] H. Zaidi and K. Koral, "Scatter modeling and compensation in emission tomography," Eur. J. Nucl. Med. Mol. Imaging, vol. 31, pp. 761-782, 2004.

[2] A. Frey, B. M. W. Tsui, and M. Ljungberg, "A comparison of scatter compensation methods in SPECT: Subtraction based techniques versus iterative reconstruction with accurate modeling of the scatter response," in Proc. IEEE Nuclear Science Symp. Medical Imaging Conf., 1993, pp. 1035-1037.
[3] F. J. Beekman, C. Kamphuis, and E. C. Frey, "Scatter compensation methods in 3-D iterative SPECT reconstruction: A simulation study," Phys. Med. Biol., vol. 42, pp. 1619-1632, 1997.

[4] C. E. Floyd, R. J. Jaszczak, K. L. Greer, and R. E. Coleman, "Inverse Monte Carlo as a unified reconstruction algorithm for ECT," J. Nucl. Med., vol. 27, pp. 1577-1585, 1986.

[5] F. J. Beekman, W. A. M. de Jong, and S. van Geloven, "Efficient fully 3-D iterative SPECT reconstruction with Monte Carlo based scatter compensation," IEEE Trans. Med. Imag., vol. 21, no. 8, pp. 867-877, Aug. 2002.

[6] A. Cot, C. Falcon, J. Pavia, F. Calvino, and D. Ros, "Evaluation of scattering models in brain SPECT imaging," in Proc. IEEE Nuclear Science Symp., vol. 4, Oct. 19-25, 2003, pp. 2901-2904.

[7] I. Buvat, D. Lazaro, and V. Breton, "Fully 3D Monte Carlo reconstruction in SPECT: Proof of concept and is it worthwhile?", presented at the Conf. 7th Int. Meeting on Fully Three-Dimensional Image Reconstruction in Radiology and Nuclear Medicine, Saint-Malo, France, Jun. 2003.

[8] K. F. Koral et al., "Initial results for hybrid SPECT-conjugate-view tumor dosimetry in I-anti-B1-antibody therapy of previously-untreated lymphoma patients," J. Nucl. Med., vol. 41, pp. 1579-1586, 2000.

[9] M. Ljungberg and S.-E. Strand, "A Monte Carlo program simulating scintillation camera imaging," Comput. Meth. Progr. Biomed., vol. 29, pp. 257-272, 1989.

[10] Y. K. Dewaraja, M. Ljungberg, and K. F. Koral, "Accuracy of I-131 tumor quantification in radioimmunotherapy using SPECT imaging with an ultra-high-energy collimator: Monte Carlo study," J. Nucl. Med., vol. 41, pp. 1760-1767, 2000.

[11] - "Characterization of scatter and penetration using Monte Carlo simulation in I-131 imaging," J. Nucl. Med., vol. 41, pp. 123-130, 2000.

[12] K. Ogawa, Y. Harata, T. Ichihara, A. Kubo, and S. Hashimoto, "A practical method for position dependent compton scatter compensation in single photon emission CT,' IEEE Trans. Med. Imag., vol. 10, no. 3, pp. 408-412, Sep. 1991.

[13] D. J. Kadrmas, E. C. Frey, S. K. Seemeen, and B. M. W. Tsui, "Fast implementations of reconstruction-based scatter compensation in fully $3 \mathrm{D}$ SPECT image reconstruction," Phys. Med. Biol., vol. 43, pp. 857-873, 1998.

[14] J. Bowsher et al., "Bayesian reconstruction and use of anatomical a priori information for emission tomography," IEEE Trans. Med. Imag., vol. 15, no. 5, pp. 673-686, Oct. 1996.

[15] M. A. King, D. J. deVries, T. S. Pan, P. H. Pretorius, and J. A. Case, "An investigation of the filtering of TEW scatter estimates used to compensate for scatter with ordered subset reconstructions," IEEE Trans. Nucl. Sci., vol. 44, no. 3, pp. 1140-1145, Jun. 1997.

[16] I. G. Zubal, C. R. Harrell, E. O. Smith, Z. Rattner, G. Gindi, and P. B. Hoffer, "Computerized three-dimensional segmented human anatomy," Med. Phys., vol. 21, pp. 299-302, 1994.

[17] Y. K. Dewaraja, S. J. Wilderman, M. Ljungberg, K. F. Koral, K. Zasadny, and M. Kaminiski, "Accurate dosimetry in I-131 radionuclide therapy using patient specific, 3-dimensional methods for SPECT reconstruction and absorbed dose calculation," J. Nucl. Med., vol. 46, pp. 840-849, 2005.

[18] M. Ljungberg, A. Larsson, and L. Johansson, "A new collimator simulation in SIMIND based on the Delta scattering technique," IEEE Trans. Nucl. Sci., vol. 52, no. 5, pp. 1370-1375, Oct. 2005.

[19] D. J. de Vries, S. C. Moore, R. E. Zimmerman, S. P. Mueller, B. Friedland, and R. C. Lanza, "Development and validation of a Monte Carlo simulation of photon transport in an anger camera," IEEE Trans. Med. Imag., vol. 9, no. 4, pp. 430-438, Dec. 1990. 